\title{
Enteral Nutrition Combined with Improved-Sijunzi Decoction Shows Positive Effect in Precachexia Cancer Patients: A Retrospective Analysis
}

\author{
Yueying Li $\mathbb{C}^{1,2,3,4}$ Yajun Chen, ${ }^{1,2,3}$ Yaqi Zeng, ${ }^{1,2,3}$ Jie Dong, ${ }^{1,2,3}$ Chunlei Li, ${ }^{1,2,3}$ Yingjie Jia, ${ }^{4}$ \\ Yonghua Zhao, ${ }^{5}$ and Kun Wang $\mathbb{C}^{1,2,3}$ \\ ${ }^{1}$ Department of Nutrition Therapy, Tianjin Medical University Cancer Institute and Hospital, \\ National Clinical Research Center for Cancer, Tianjin, China \\ ${ }^{2}$ Key Laboratory of Cancer Prevention and Therapy, Tianjin, China \\ ${ }^{3}$ Tianjin's Clinical Research Center for Cancer, Huanhu West Road, Hexi District, Tianjin 300060, China \\ ${ }^{4}$ Department of Oncology, First Teaching Hospital of Tianjin University of Traditional Chinese Medicine, \\ No. 88 Changling Road, Liqizhuang Street, Xiqing District, Tianjin 300380, China \\ ${ }^{5}$ State Key Laboratory of Quality Research in Chinese Medicine, Institute of Chinese Medical Sciences, University of Macau, \\ Macau SAR, Taipa 999078, China
}

Correspondence should be addressed to Kun Wang; zlyywangkun@163.com

Received 18 May 2021; Revised 31 August 2021; Accepted 8 September 2021; Published 24 September 2021

Academic Editor: Yoshiki Mukudai

Copyright (C) 2021 Yueying Li et al. This is an open access article distributed under the Creative Commons Attribution License, which permits unrestricted use, distribution, and reproduction in any medium, provided the original work is properly cited.

Background. Cancer has been considered as the leading cause of death in the world. In patients with cancer, up to $80 \%$ display a cachectic period after diagnosis. Cachexia is known to have a negative impact on function, treatment tolerance, higher rates of hospitalizations, and mortality. Anorexia is often used as a warning sign of precachexia. Long-term anorexia may lead to malnutrition and, then, accelerate the occurrence of cachexia. A safe and effective treatment, which can both improve appetite and assist nutritional support for precachexia cancer patients shows its particular important role. Methods. A retrospective analysis comparing the different therapeutic effects on precachexia cancer patients with anorexia-malnutrition. We recorded 46 patients with the improved-Sijunzi decoction combined with enteral nutrition emulsion (ISJZ group) and 35 patients with single enteral nutrition emulsion (SEN group). The different therapeutic effects of the two groups were observed by recording indicators before and 2 weeks after treatment, including patient-generated subjective global assessment score, quality of life score, Karnofsky performance status scale, Eastern cooperative oncology group scale standard and traditional Chinese medicine syndrome, daily total dietary intake, red blood cells, hemoglobin, prealbumin, albumin, total protein cholinesterase, C-reactive protein, leukocytes, alanine aminotransferase, aspartate aminotransferase, alkaline phosphatase, urea nitrogen, and creatinine. Results. ISJZ group exhibited prominent improvement of traditional Chinese medicine syndrome (TCMS), nutritional condition, and quality of life compared with the SEN group (QOL: $p=0.0001$, PG-SGA: $p=0.019$, dietary intake: $p=0.0001$, TCMS: $p=0.0001$ ). The levels of HGB $(p=0.006)$, PAlb $(p=0.001)$, Alb $(p=0.0001)$, TP $(p=0.008)$, and ChE $(p=0.0001)$ in the ISJZ group were higher than the SEN group after treatment. Moreover, the ratios of CRP/ALB $(p=0.028)$ and CRP/PALB $(p=0.005)$ in the two groups have obvious differences; they were lower for the ISJZ group than the SEN group. Conclusions. Enteral nutrition combined with ISJZ decoction is an effective treatment in precachexia cancer patients for the prevention of cachexia. This treatment therapy can alleviate the inflammatory response, improve malnutrition state, and promote the performance status. Tianjin Medical University Cancer Institute and Hospital approved this study (Trial No. 1913). 


\section{Introduction}

Cancer has been considered as the leading cause of death in the world $[1,2]$. Anorexia is one of the top three common symptoms in patients with cancer $[3,4]$, and it may induce malnutrition. The appearance of anorexia is often used as a warning sign of precachexia. It can be an independent prognostic factor for survival [5]. In patients with cancer, up to $80 \%$ display a cachectic period after diagnosis [6-8]. Cachexia is known to have a negative impact on function, treatment tolerance, higher rates of hospitalizations, and mortality $[9,10]$. The development process of cachexia can be fast or slow, but it will all start from precachexia to refractory cachexia [7]; however, the reversal of refractory cachexia cannot be achieved at present. Hence, we should intervene in anorexia-malnutrition as soon as possible [11]. The treatment of anorexia-malnutrition includes invasive/parenteral nutrition support, enteral nutrition support, and appetite stimulants. However, we are still not sure which is the best time to offer artificial nutrition during cancer treatment [12]. What's more, some reports advised considering enteral and parenteral feeding only when the prognosis is longer than months to weeks [13]. The most extensively and successfully studied appetite stimulants are progestational agents and corticosteroids. Unfortunately, they all present side effects with varying degrees, such as the potential serious adverse events of megestrol acetate including thrombophlebitis [14] and adrenal insufficiency after abrupt drug withdrawal. Long-term steroids may induce metabolic changes, higher fracture rates, cataracts, and gastrointestinal discomfort [11]. Anamorelin is an oral ghrelin mimetic, and it has been proven to improve body weight. However, its drug toxicities included hyperglycemia and gastrointestinal disorders [15]. Therefore, we try to find a safe and effective treatment that can both improve appetite and assist nutritional support.

Sijunzi (SZJ) decoction comes from the Taiping Huimin and the Agent Bureau [16]. SJZ decoction has the effect of replenishing qi and invigorating spleen. It is used to treat the TCM syndrome of spleen qi deficiency. The main clinical manifestations of "spleen qi deficiency" include decreasing appetite, deficiency of food, debilitation, and abdominal distension (intermittently) [17, 18]. SJZ decoction could improve the immune function and QOL. What's more, SZJ decoction could maintain peripheral blood leukocyte count and peritoneal macrophage activity after chemotherapy; in addition, it could regulate the immune function of the body and enhance the anticancer effect of immune cells [19-22]. SJZ decoction could inhibit gastric cancer cell growth in vivo, and the mechanism was associated with the downregulation of the expression of p53 mRNA [23]. Chen et al. reported SJZ decoction produced anti-H22 tumor cells, upregulated the level of Bax, caspase-3, TNF- $\alpha$, and IL-2, and downregulated Bcl-2 [24]. Dai et al. provided strong evidence for treating chronic fatigue syndrome by using SJZ decoction [25]. We improved the SJZ decoction to make it suitable for cancer patients with anorexia-malnutrition with spleen qi deficiency syndrome.
Some cancer patients with anorexia-malnutrition conform to the TCM syndrome of spleen qi deficiency syndrome. Long-term anorexia-malnutrition may lead to cachexia. In order to avoid the occurrence of irreversible cachexia, a safe and effective treatment urgently needs to be discovered, so we explored whether ISJZ decoction could have a positive effect on such patients. This passage reports the positive role of ISJZ in improving anorexia-malnutrition patients with spleen qi deficiency.

\section{Materials and Methods}

2.1. Study Population. We retrospectively reviewed the records of 81 patients between October 2018 and August 2020 in Tianjin Medical University Cancer Institute and Hospital. The study was conducted in accordance with the Declaration of Helsinki (as revised in 2013). The study was approved by ethics committee of Tianjin Medical University Cancer Institute and Hospital (No. E2019310). This study is a retrospective study and an informed consent form was not required. Inclusion criteria: (1) had pathologically diagnosed cancer; (2) age between 18-80 years old; (3) expected survival time was more than 3 months; (4) PG-SGA (patientgenerated subjective global assessment) score $\geq 4$ points; (5) 10 points $<$ KPS (Karnofsky performance status) score $\leq 90$ points; (6) 1 points $\leq$ ECOG (Eastern cooperative oncology group) score $\leq 4$ points; (7) corresponded TCM syndromes of spleen qi deficiency; (8) corresponded the definition of "precachexia" [26]; and (9) patients accepted treatment by enteral nutritional support or ISJZ decoction combined with enteral nutritional support. Exclusion criteria: (1) need parenteral nutrition treatment; (2) ongoing anticancer treatment; (3) taking other Chinese herbal medicines or Chinese patent medicine in one week; (4) taking other drugs or blood products for treatment of malnutrition in one week; (5) had history of anti-infection therapies in one week; (6) had treatments that affected immune indicators; (7) taking appetite stimulants; (8) numeric pain rating scale score $\geq 7$ points; (9) serious dysfunction of heart, lungs, brain, liver, or kidney; (10) confirmed state of refractory cachexia; and (11) disturbance of consciousness, dementia, or depression. We divided 81 patients into two groups according to the different treatment therapies, ISJZ group $(n=46)$ and SEN group $(n=35)$.

2.2. Assessments. We recorded the gender and age of each group of patients. The PG-SGA score, QOL score [27], KPS scale, ECOG scale standard, and TCM syndrome score $[28,29]$ (see the scoring method of traditional Chinese medicine syndrome in Table 1) were used to evaluate patients' subjective feelings and performance state. Daily total dietary intake, RBC (red blood cell), HGB (hemoglobin), PAlb (prealbumin), Alb (albumin), TP (total protein), and ChE (cholinesterase) were used to evaluate patients' objective nutritional status. We analyzed CRP (C-reactive protein) and WBC (leukocyte) to judge inflammation. ALT (alanine aminotransferase), AST (aspartate aminotransferase), ALP (alkaline phosphatase), UREA (urea nitrogen), 
TABLE 1: The scoring method of traditional Chinese medicine syndrome.

\begin{tabular}{|c|c|c|c|}
\hline Symptom & 1 score & 2 score & 3 score \\
\hline $\begin{array}{l}\text { Decreased food } \\
\text { intake }\end{array}$ & Less than $1 / 3$ & Between $1 / 3$ and $1 / 2$ & More than $1 / 2$ \\
\hline $\begin{array}{l}\text { Lack of strength } \\
\text { Abdominal } \\
\text { distention }\end{array}$ & $\begin{array}{l}\text { Unable to adhere manual labor } \\
\text { Mild fullness, abdominal } \\
\text { distension after eating, relieved } \\
\text { within half an hour. }\end{array}$ & $\begin{array}{l}\text { Barely stick to daily activities } \\
\text { Abdominal distension, obvious after } \\
\text { eating, relieved within half an hour to } \\
1 \text { hour. }\end{array}$ & $\begin{array}{l}\text { Unable to adhere to daily activities } \\
\text { Abdominal distension is obvious, } \\
\text { especially after eating, relieving takes } \\
\text { more than } 1 \text { hour. }\end{array}$ \\
\hline $\begin{array}{l}\text { Constipation or } \\
\text { loose stools }\end{array}$ & & 1 score & \\
\hline
\end{tabular}

TCMS score was the sum of each symptom score.

and CREA (creatinine) were used to evaluate drug safety. All indicators were recorded before and 2 weeks after treatment to assess whether ISJZ decoction can have a positive effect on patients.

2.3. Treatment. SEN group: patients in this group were given enteral nutritional support to supply the gap of energy requirement (the gap of energy $\mathrm{kcal}=30 \mathrm{kcal} \times$ weight $(\mathrm{kg})$ dietary intake (kcal)). Enteral nutrition emulsion TPF $T$ $(1.3 \mathrm{kcal} / \mathrm{ml})$ was supplemented according to the calculation results for each patient.

ISJZ group: patients in this group were given ISJZ decoction, additionally, which consists of Codonopsis (dangshen) $30 \mathrm{~g}$, rhizoma atractylodes (baizhu) $10 \mathrm{~g}$, Poria cocos (fuling) $10 \mathrm{~g}$, Radix glycyrrhizae (gancao) $6 \mathrm{~g}$, Astragalus (huangqi) $30 \mathrm{~g}$, Radix curcuma (jianghuang) $10 \mathrm{~g}$, jiao Crataegus (shanzha) $10 \mathrm{~g}$, jiao Medicated Leaven (shenqu) $10 \mathrm{~g}$, and Ziziphus jujube (dazao) $10 \mathrm{~g}$. Each component was processed into granules from Jiangyin Tianjiang Pharmaceutical Co, Ltd. (China). The patients were required to take the ISJZ granules twice a day (intake: mixed with hot water, $300 \mathrm{ml}$ daily, taken morning and evening, $150 \mathrm{ml}$ each time). The basic nutrition treatment was the same as that of the SEN group.

2.4. Statistical Analysis. SPSS software (Version 23.0, SPSS Inc, Chicago, IL, USA) was used for statistical analysis. The Shapiro-Wilk test was used to detect whether the data conform to the normal distribution. Normally distributed data were expressed as mean \pm standard deviation ( $X \pm \mathrm{SD})$, using the $T$ test to compare two groups of data. Abnormal distributed data were expressed as median (p25, p75), using Mann-Whitney $U$ test to compare the two groups of data. The categorical data were expressed as ratios (\%), using Chi-square tests to compare between groups. $p<0.05$ (two-sided) was recorded as a statistically significant difference between the two groups.

\section{Results}

3.1. Characteristics of Patients. A total of 81 patients were included in our study, and all of them belong to clinical stage IV. Table 2 detailed the cancer primary site of 2 groups of patients. Patients in 2 groups had similar age $(58.7 \pm 11.3$ vs. $63.1 \pm 9.8$ ) and sex (male: $52.17 \%$ vs. $60 \%$ ) composition, and there was no statistical difference in the type of previous treatment $(1.98 \pm 0.99$ vs. $2.15 \pm 1.18$; previous treatment included operation, radiotherapy, chemotherapy, hormonal treatment, targeted therapy, and immunotherapy). However, the analysis showed that the number of distant metastasis between the two groups was statistically significant $(p=0.026)$. In the comparison of the baseline nutritional status, there were no significant differences in PG-SGA, QOL, KPS, ECOG, and TCMS scores between the two groups (Table 2).

3.2. Performance Status, Nutritional Condition, and Quality of Life Assessment. Before treatment, patients in the two groups were in high nutritional risk and urgently needed proper nutritional support. After treatment, the nutritional condition of the patients had improved to varying degrees in the two groups. The change in the PG-SGA score and TCMS score in the ISJZ group was decreased more than that in SEN group. The change in the dietary intake and QOL score in the ISJZ group was increased more than that in the SEN group, they exhibited a significant difference in the 2 groups ( $p=0.0001$, Table 3 ). The difference in the KPS and ECOG scores had no significant difference between the 2 groups. The results demonstrated that enteral nutrition could improve patients' quality of life and alleviate clinical symptoms. This when combined with ISJZ decoction could amplify the effect.

3.3. Hematological Examination. Next, we compared the changes in hematologic examination before and after the treatment of patients in the 2 groups (Table 4 ). The analysis showed that the RBC, HGB, PAlb, Alb, TP, and ChE levels were increased at the end of treatment in the ISJZ group. However, the RBC, HGB, PAlb, Alb, and TP levels were decreased at the end of treatment in the SEN group. There was no difference in HGB, PAlb, Alb, TP, and ChE levels between the two groups before treatment, but there was a significant difference at the end of treatment $(p=0.006, p=0.001, p=0.0001, p=0.008, p=0.0001)$ and the difference was statistically significant $(p=0.0001$, $p=0.0001, p=0.0001, p=0.0001, p=0.043)$.

Furthermore, the analysis showed that the CRP level was decreased at the end of treatment in two groups. The levels of $\mathrm{CRP} / \mathrm{Alb}$ and CRP/PAlb were decreased in the ISJZ group; however, they were increased in the SEN group at the end of 
TABLE 2: Characteristic of patients.

\begin{tabular}{|c|c|c|c|}
\hline Characteristic & ISJZ group $(n=46), n(\%)$ & SEN group $(n=35), n(\%)$ & $p$-value \\
\hline Male & $24(52.17)$ & $21(60)$ & 0.221 \\
\hline Female & $22(47.83)$ & $14(40)$ & \\
\hline Age (years) & $58.7 \pm 11.3$ & $63.1 \pm 9.8$ & 0.388 \\
\hline PG-SGA score & $15.48 \pm 5.16$ & $15.23 \pm 4.37$ & 0.819 \\
\hline QOL score & $25.26 \pm 4.07$ & $23.31 \pm 6.1$ & 0.089 \\
\hline KPS score & $59.13 \pm 8.90$ & $60.29 \pm 7.47$ & 0.537 \\
\hline ECOG score & $2.02 \pm 0.61$ & $2.11 \pm 0.63$ & 0.509 \\
\hline TCMS score & $5.74 \pm 1.45$ & $5.86 \pm 1.35$ & 0.710 \\
\hline \multicolumn{4}{|c|}{ Primary site } \\
\hline Bile duct & 1 & 0 & - \\
\hline Bladder & 1 & 1 & - \\
\hline Breast & 4 & 4 & - \\
\hline Cervix & 4 & 3 & - \\
\hline Esophagus & 5 & 6 & - \\
\hline Kidney & 1 & 1 & - \\
\hline Liver & 1 & 1 & - \\
\hline Lung & 18 & 7 & - \\
\hline Ovaries & 2 & 0 & - \\
\hline Pancreas & 3 & 3 & - \\
\hline Prostate & 0 & 3 & - \\
\hline Rectum & 1 & 2 & - \\
\hline Stomach & 0 & 2 & - \\
\hline Ureter & 0 & 1 & - \\
\hline Others & 5 & 1 & - \\
\hline Number of distant metastasis & $2.02 \pm 1.14$ & $1.56 \pm 0.66$ & $0.026^{*}$ \\
\hline Type of previous treatment & $1.98 \pm 0.99$ & $2.15 \pm 1.18$ & 0.293 \\
\hline
\end{tabular}

*, statistical significance at $p<0.05$. PG-SGA, patient-generated subjective global assessment; QOL, quality of life; KPS, Karnofsky performance status; ECOG, Eastern cooperative oncology group; TCMS: traditional Chinese medicine syndrome.

TABle 3: Comparison of patients' quality of life and nutritional status.

\begin{tabular}{|c|c|c|c|c|}
\hline Survey score & Time & ISJZ group & SEN group & $p$-value \\
\hline PG-SGA score & $\begin{array}{c}\text { Baseline } \\
\text { Ending } \\
\text { Difference }\end{array}$ & $\begin{array}{c}15.48 \pm 5.16 \\
7.33 \pm 2.16 \\
-8.50(-11.25,-4.75) \\
\end{array}$ & $\begin{array}{c}15.23 \pm 4.37 \\
13.09 \pm 3.88 \\
-2.50(-2.75,0.00) \\
\end{array}$ & $\begin{array}{c}0.819 \\
0.019^{*} \\
0.0001^{* *} \\
\end{array}$ \\
\hline Dietary intake (kcal) & $\begin{array}{c}\text { Baseline } \\
\text { Ending } \\
\text { Difference }\end{array}$ & $\begin{array}{c}980 \pm 113 \\
1284 \pm 122 \\
293.00(208.50,453.50)\end{array}$ & $\begin{array}{c}1014 \pm 123 \\
1017 \pm 92 \\
31.00(-90.00,125.00)\end{array}$ & $\begin{array}{c}0.198 \\
0.0001^{* *} \\
0.0001^{* *} \\
\end{array}$ \\
\hline QOL score & $\begin{array}{c}\text { Baseline } \\
\text { Ending } \\
\text { Difference }\end{array}$ & $\begin{array}{c}25.26 \pm 4.07 \\
37.04 \pm 3.15 \\
11.00(9.75,14.00) \\
\end{array}$ & $\begin{array}{c}23.31 \pm 6.1 \\
28.40 \pm 4.68 \\
6.00(3.00,7.00) \\
\end{array}$ & $\begin{array}{c}0.089 \\
0.0001^{* *} \\
0.0001^{* *} \\
\end{array}$ \\
\hline KPS score & $\begin{array}{c}\text { Baseline } \\
\text { Ending } \\
p \text {-value*** } \\
\text { Difference }\end{array}$ & $\begin{array}{c}59.13 \pm 8.90 \\
62.17 \pm 5.93 \\
0.0001^{* *} \\
0.00(0.00,10.00) \\
\end{array}$ & $\begin{array}{c}60.29 \pm 7.47 \\
61.43 \pm 6.48 \\
0.074 \\
0.00(0.00,0.00) \\
\end{array}$ & $\begin{array}{l}0.537 \\
0.592 \\
0.093 \\
\end{array}$ \\
\hline ECOG score & $\begin{array}{c}\text { Baseline } \\
\text { Ending } \\
p \text {-value** } \\
\text { Difference }\end{array}$ & $\begin{array}{c}2.02 \pm 0.61 \\
1.87 \pm 0.45 \\
0.003^{*} \\
0.00(0.00,0.00) \\
\end{array}$ & $\begin{array}{c}2.11 \pm 0.63 \\
2.00 \pm 0.59 \\
0.659 \\
0.00(0.00,0.00) \\
\end{array}$ & $\begin{array}{l}0.509 \\
0.265 \\
0.686 \\
\end{array}$ \\
\hline TCMS score & $\begin{array}{l}\text { Baseline } \\
\text { Ending } \\
\text { Difference }\end{array}$ & $\begin{array}{c}5.74 \pm 1.45 \\
4.22 \pm 1.07 \\
-1.00(-2.00,-1.00)\end{array}$ & $\begin{array}{c}5.86 \pm 1.35 \\
5.26 \pm 1.04 \\
-1.00(-1.00,-1.00)\end{array}$ & $\begin{array}{c}0.710 \\
0.0001^{* *} \\
0.01^{*}\end{array}$ \\
\hline
\end{tabular}

${ }^{*}$ Statistical significance at $p<0.05$; ${ }^{* *}$ statistical significance at $p<0.001$. Baseline and ending: mean \pm SD; difference: median (p25, p50). ${ }^{* * *} p$-value between baseline and ending in the same group. PG-SGA, patient enerated subjective global assessment; QOL, quality of life; KPS, Karnofsky performance status; ECOG, Eastern Cooperative Oncology Group; TCMS: traditional Chinese medicine syndrome. 
TABLE 4: Comparison of patients' blood indicators.

\begin{tabular}{|c|c|c|c|c|}
\hline Blood indicator & Time & ISJZ group & SEN group & $p$-value \\
\hline \multirow{3}{*}{$\mathrm{RBC}(1012 / \mathrm{L})$} & Baseline & $3.54 \pm 0.60$ & $3.98 \pm 0.69$ & $0.004^{*}$ \\
\hline & Ending & $3.81 \pm 0.58$ & $3.55 \pm 0.76$ & 0.092 \\
\hline & Difference & $0.21(0.10,0.45)$ & $-0.35(-0.62,-0.18)$ & $0.0001^{* *}$ \\
\hline \multirow{3}{*}{ HGB (g/L) } & Baseline & $106.48 \pm 16.47$ & $114.60 \pm 20.36$ & 0.051 \\
\hline & Ending & $114.17 \pm 17.43$ & $101.91 \pm 20.50$ & $0.006^{*}$ \\
\hline & Difference & $7.00(2.00,12.00)$ & $-10.00(-20.00,-5.00)$ & $0.0001^{* *}$ \\
\hline \multirow{3}{*}{ PAlb (g/L) } & Baseline & $0.14 \pm 0.05$ & $0.14 \pm 0.07$ & 0.638 \\
\hline & Ending & $0.19 \pm 0.07$ & $0.13 \pm 0.07$ & $0.001^{* *}$ \\
\hline & Difference & $0.05(0.02,0.083)$ & $-0.02(-0.07,0.06)$ & $0.0001^{* *}$ \\
\hline \multirow{3}{*}{ Alb (g/L) } & Baseline & $32.63 \pm 6.89$ & $35.44 \pm 9.24$ & 0.120 \\
\hline & Ending & $36.22 \pm 5.04$ & $30.24 \pm 6.64$ & $0.0001^{* *}$ \\
\hline & Difference & $2.85(0.70,4.90)$ & $-2.6(7.05,0.13)$ & $0.0001^{* *}$ \\
\hline \multirow{3}{*}{$\mathrm{TP}(\mathrm{g} / \mathrm{L})$} & Baseline & $61.15 \pm 6.96$ & $66.76 \pm 7.21$ & 0.053 \\
\hline & Ending & $67.13 \pm 7.15$ & $62.06 \pm 8.97$ & $0.008^{*}$ \\
\hline & Difference & $4.95(2.65,7.68)$ & $-4.90(-9.50,3.50)$ & $0.0001^{* *}$ \\
\hline \multirow{3}{*}{$\operatorname{ChE}(\mathrm{U} / \mathrm{L})$} & Baseline & $5671.21 \pm 1708.04$ & $4930.71 \pm 2053.72$ & 0.081 \\
\hline & Ending & $6340.07 \pm 1594.36$ & $4776.43 \pm 1893.46$ & $0.0001^{* *}$ \\
\hline & Difference & $576.50(93.50,890.50)$ & $61.00(-2055.00,827.00)$ & $0.043^{*}$ \\
\hline \multirow{3}{*}{ CRP (mg/L) } & Baseline & 42.75 & 37.80 & 0.535 \\
\hline & Ending & 19.75 & 38.10 & 0.081 \\
\hline & Difference & $-10.27(-45.99,2.38)$ & $-3.00(-41.60,14.00)$ & 0.308 \\
\hline \multirow{3}{*}{$\mathrm{CRP} / \mathrm{Alb}$} & Baseline & $1.32(0.28,2.69)$ & $0.96(0.52,2.94)$ & 0.696 \\
\hline & Ending & $0.57(0.14,1.67)$ & $1.52(0.41,3.16)$ & $0.028^{*}$ \\
\hline & Difference & $-0.38(-1.76,0.014)$ & $0.045(-0.92,1.32)$ & $0.027^{*}$ \\
\hline \multirow{3}{*}{$\mathrm{CRP} / \mathrm{PAlb}$} & Baseline & $0.33(0.07,0.93)$ & $0.46(0.10,1.04)$ & 0.443 \\
\hline & Ending & $0.11(0.03,0.34)$ & $0.38(0.11,0.94)$ & $0.005^{*}$ \\
\hline & Difference & $-0.14(-0.58,-0.00)$ & $0.02(-0.42,0.37)$ & $0.032^{*}$ \\
\hline \multirow{3}{*}{ WBC $(109 / \mathrm{L})$} & Baseline & $7.40 \pm 3.61$ & $9.06 \pm 4.37$ & 0.065 \\
\hline & Ending & $7.55 \pm 3.45$ & $8.21 \pm 3.80$ & 0.413 \\
\hline & Difference & $0.56(-0.33,1.92)$ & $-0.80(-2.24,0.60)$ & $0.01^{*}$ \\
\hline \multirow{3}{*}{$\operatorname{ALT}(\mathrm{U} / \mathrm{L})$} & Baseline & $19.98 \pm 15.34$ & $18.71 \pm 14.60$ & 0.709 \\
\hline & Ending & $27.32 \pm 17.42$ & $20.29 \pm 16.06$ & 0.066 \\
\hline & Difference & $3.00(-4.25,18.75)$ & $1.00(-13.00,11.00)$ & 0.256 \\
\hline \multirow{3}{*}{ AST (U/L) } & Baseline & $24.54 \pm 13.58$ & $30.60 \pm 25.02$ & 0.167 \\
\hline & Ending & $29.54 \pm 15.81$ & $32.86 \pm 32.86$ & 0.551 \\
\hline & Difference & $2.50(-3.50,12.25)$ & $-2.00(-14.00,17.00)$ & 0.190 \\
\hline \multirow{3}{*}{ ALP (U/L) } & Baseline & $125.74 \pm 76.38$ & $154.09 \pm 114.33$ & 0.185 \\
\hline & Ending & $140.48 \pm 79.65$ & $189.91 \pm 206.49$ & 0.141 \\
\hline & Difference & $8.00(-3.75,24.50)$ & $3.00(-64.00,73.00)$ & 0.706 \\
\hline \multirow{3}{*}{ CREA $(\mu \mathrm{mol} / \mathrm{L})$} & Baseline & $55.93 \pm 11.97$ & $56.51 \pm 16.77$ & 0.856 \\
\hline & Ending & $52.56 \pm 11.80$ & $51.34 \pm 16.63$ & 0.704 \\
\hline & Difference & $-1.00(-7.5,1.50)$ & $-5.00(-24.00,8.00)$ & 0.464 \\
\hline \multirow{3}{*}{ UREA (mmol/L) } & Baseline & $4.52 \pm 1.48$ & $6.36 \pm 4.68$ & $0.030^{*}$ \\
\hline & Ending & $4.97 \pm 1.93$ & $5.73 \pm 2.39$ & 0.116 \\
\hline & Difference & $0.20(-0.45,1.00)$ & $-0.10(-3.10,1.70)$ & 0.167 \\
\hline
\end{tabular}

${ }^{*}$ Statistical significance at $p<0.05 ;{ }^{* *}$ statistical significance at $p<0.001$. Baseline and ending: mean \pm SD; difference: median (p25, p50). RBC, red blood cell; HGB, hemoglobin; PAlb, prealbumin; Alb, albumin; TP, total protein; ChE, cholinesterase; CRP, C-reactive protein; WBC, leukocyte; ALT, alanine aminotransferase; AST, aspartate aminotransferase; ALP, alkaline phosphatase; CREA, creatinine; UREA, urea nitrogen.

the treatment. There was no difference in CRP/Alb and CRP/ PAlb levels between the two groups before treatment, but there was a significant difference at the end of treatment ( $p=0.028, p=0.005)$ and the difference was statistically significant $(p=0.027$ and $p=0.032)$. There was no difference in the level of WBC between the two groups before and after treatment.
3.4. Evaluation of Drug Safety. We evaluated the drug safety by monitoring the liver and kidney functions. ALT, AST, ALP, CREA, and UREA were selected to observe whether the drug has liver or kidney toxicity. The degrees of ALT, AST, ALP, and CREA showed no significant differences before and after treatment in two groups. This result suggested that ISJZ decoction had no obvious liver and kidney toxicity. 


\section{Discussion}

Cancer anorexia is diagnosed on the basis of loss of appetite, and/or early satiety, taste alterations, nausea, and vomiting [30], which caused energy intake deficiency [31]. Continuous energy intake deficiency may lead to patient malnutrition, cause serious effects on patient's quality of life, and interfere with cancer treatment therapy. The formation of cachexia can be attributed to two factors: one is tumorinduced metabolic changes and another is anorexia-induced malnutrition [30, 32]. We observed peripheral blood samples as a prognostic indicator for judging patients with malignant tumors [33].

Multiple inflammatory responses play a pivotal part in tumor-induced metabolic changes. The ratio CRP/Alb is a prognostic indicator for several cancers [34]. Because of its shorter half-life, PAlb is used as a good indicator of visceral protein [35]. Some researchers suggested that CRP/PAlb was more sensitive to CRP/Alb for cancer prognosis. High CRP/ Alb or CRP/PAlb ratio was significantly associated with bad prognosis [36]. Our results showed that patients who only accepted enteral nutrition had a decreasing trend in the CRP level, but not in the ratio of $\mathrm{CRP} / \mathrm{Alb}$ and CRP/PAlb. However, the levels of CRP, CRP/Alb, and CRP/PAlb in patients who underwent treatment in combination with ISJZ decrease at the same time. So far, we have boldly assumed that ISZJ decoction could alleviate the inflammatory response triggered by cancer, so that it partly suppressed the consequence of metabolic abnormalities.

Anorexia-induced malnutrition has a negative effect on prognosis. ChE as a nutrition-related serum marker is useful for predicting the prognosis of patients with several types of cancer $[37,38]$. Patients with low levels of ChE and Alb have the lowest 5-year overall survival rates than those patients with high levels of $\mathrm{ChE}$ and Alb or high ChE/low Alb or low $\mathrm{ChE/high} \mathrm{Alb} \mathrm{[37].} \mathrm{Our} \mathrm{results} \mathrm{indicated} \mathrm{that} \mathrm{the} \mathrm{short-term}$ enteral nutritional treatment failed to increase the level of ChE or Alb. However, treatment combined with ISJZ decoction could increase the ChE level (6340.07 \pm 1594.36$)$ and the Alb (36.22 \pm 5.04$)$ level (Table 4). Our results also showed that the levels of RBC, HGB, PAlb, and TP were increased within the normal range in the ISJZ group. Additionally, the value of dietary intake in the SEN group was increased lower than that in the ISJZ group, and the score of TCMS in the SEN group was decreased lower than that in the ISJZ group. On the basis of these data, we speculated that the reason why only accepted short-term enteral nutrition could not improve the nutritional status in time might be related to its inability to improve appetite and absorption. The addition of ISJZ on the basis of nutritional therapy could effectively increase appetite and improve nutritional status, thereby might have an opportunity to increase the five-year survival rate.

As a predicted survival in patients with cancer, the performance status (PS) had the ability to measure the activities of daily living. It is well known that patients with poor performance status always avoid more aggressive treatment [39-41]. The KPS score and ECOG score are the most commonly used for the assessment of PS. Generally speaking, the KPS score is required no less than 70, and the ECOG score is required no more than 2 before relevant anticancer treatments are considered. We recorded two groups of patients, KPS and ECOG scores. Our results showed that KPS score was less than 70 and ECOG score was more than 2 in both groups before treatment. It is demonstrated that the conditions of patients were suggested not to tolerate more aggressive anticancer treatment. After treatment, KPS scores of the two groups were all increased, and the ECOG scores of the two groups were all decreased. Regrettably, we could not find that ISJZ had a significant advantage than SEN in a short treatment period. However, it is worth noting that the score of KPS and ECOG in the ISJZ group had significant differences before and after treatment, especially the score of ECOG decreased to lower than $2(1.87 \pm 0.45$, Table 3$)$. The TCMS score of the ISJZ group was significantly lower than that of the SEN group ( $p=0.0001$, Table 3$)$, the QOL score of the ISJZ group was significantly higher than that in the SEN group $(p=0.0001$, Table 3), and the differences were statistically significant after treatment. Hence, we thought that ISJZ decoction can promote the physical recovery of patients, reduce the clinical symptoms of patients, and reduce the suffering of patients.

Our study has certain limitations. First, the study was not a double-blind random trial. Second, the term of observation was short, so we could not give the evaluation of long-term effects. Third, our current evidence could only show that ISJZ decoction was suitable for patients with spleen-qi deficiency. Nevertheless, our results gave strong evidence for the possibility that enteral nutrition combined with ISJZ decoction was efficacious and safe for improving precachexia in cancer patients with spleen qi deficiency.

\section{Conclusions}

Our findings suggested that the treatment of enteral nutrition combined with ISJZ decoction could (i) alleviate the inflammatory response triggered by cancer, (ii) improve the malnutrition state triggered by anorexia, and (iii) promote patient's performance status.

\section{Abbreviations}

Alb: Albumin

ALP: Alkaline phosphatase

ALT: Alanine aminotransferase

AST: Aspartate aminotransferase

ChE: Cholinesterase

CRP: c-Reactive protein

CREA: Creatinine

ECOG: Eastern Cooperative Oncology Group

HGB: Hemoglobin

KPS: Karnofsky performance status

PAlb: Prealbumin

PG-SGA: Patient-generated subjective global assessment

PS: $\quad$ Performance status

QOL: Quality of life

RBC: $\quad$ Red blood cells

TCMS: Traditional Chinese medicine syndrome 
TP: $\quad$ Total protein

UREA: Urea nitrogen

WBC: Leukocyte.

\section{Data Availability}

The data in this study are available on request from the corresponding author. The data are not publicly available due to patient privacy.

\section{Consent}

This study is a retrospective analysis, and informed consent form was not required. However, all treatment and assessment data received by patients were reasonable and consensual.

\section{Conflicts of Interest}

The authors declare no conflicts of interest.

\section{Acknowledgments}

This research was funded by Tianjin Medical University Cancer Institute and Hospital, Tumor Translational Medicine Seed Fund Project (grant no. 1913).

\section{References}

[1] F. Bray, J. Ferlay, I. Soerjomataram, R. L. Siegel, L. A. Torre, and A. Jemal, "Global cancer statistics 2018: GLOBOCAN estimates of incidence and mortality worldwide for 36 cancers in 185 countries," CA: A Cancer Journal for Clinicians, vol. 68, no. 6, pp. 394-424, 2018.

[2] J. Ferlay, M. Colombet, I. Soerjomataram et al., "Estimating the global cancer incidence and mortality in 2018: GLOBOCAN sources and methods," International Journal of Cancer, vol. 144, no. 8, pp. 1941-1953, 2019.

[3] J. M. Giesinger, L. M. Wintner, A. S. Oberguggenberger et al., "Quality of life trajectory in patients with advanced cancer during the last year of life," Journal of Palliative Medicine, vol. 14, no. 8, pp. 904-912, 2011.

[4] B. B. Reeve, S. A. Mitchell, A. C. Dueck et al., "Recommended patient-reported core set of symptoms to measure in adult cancer treatment trials," Journal of the National Cancer Institute, vol. 106, 2014.

[5] F. Zhang, A. Shen, Y. Jin, and W. Qiang, "The management strategies of cancer-associated anorexia: a critical appraisal of systematic reviews," BMC Complementary and Alternative Medicine, vol. 18, no. 1, p. 236, 2018.

[6] A. Inui, "Cancer anorexia-cachexia syndrome: current issues in research and management," CA: A Cancer Journal for Clinicians, vol. 52, no. 2, pp. 72-91, 2002.

[7] K. Fearon, F. Strasser, S. D. Anker et al., "Definition and classification of cancer cachexia: an international consensus," The Lancet Oncology, vol. 12, no. 5, pp. 489-495, 2011.

[8] Y. Belloum, F. Rannou-Bekono, and F. B. Favier, "Cancerinduced cardiac cachexia: pathogenesis and impact of physical activity," Oncology Reports, vol. 37, no. 5, pp. 2543-2552, 2017.

[9] P. Kasvis, M. Vigano, and A. Vigano, "Health-related quality of life across cancer cachexia stages," Annals of Palliative Medicine, vol. 8, no. 1, pp. 33-42, 2019.
[10] J. Farkas, S. Von Haehling, K. Kalantar-Zadeh, J. E. Morley, S. D. Anker, and M. Lainscak, "Cachexia as a major public health problem: frequent, costly, and deadly," Journal of Cachexia, Sarcopenia and Muscle, vol. 4, no. 3, pp. 173-178, 2013.

[11] D. S. Childs and A. Jatoi, "A hunger for hunger: a review of palliative therapies for cancer-associated anorexia," Annals of Palliative Medicine, vol. 8, no. 1, pp. 50-58, 2019.

[12] J. Arends, P. Bachmann, V. Baracos et al., "ESPEN guidelines on nutrition in cancer patients," Clinical Nutrition, vol. 36, no. 1, pp. 11-48, 2017.

[13] M. Dans, T. Smith, A. Back et al., "NCCN guidelines insights: palliative care, version 2.2017," Journal of the National Comprehensive Cancer Network, vol. 15, no. 8, pp. 989-997, 2017.

[14] V. Ruiz Garcia, E. López-Briz, R. Carbonell Sanchis, J. L. Gonzalvez Perales, and S. Bort-Marti, "Megestrol acetate for treatment of anorexia-cachexia syndrome," Cochrane Database of Systematic Reviews, vol. 2013, Article ID Cd004310, 2013.

[15] G. Fonseca and S. Von Haehling, "An overview of anamorelin as a treatment option for cancer-associated anorexia and cachexia," Expert Opinion on Pharmacotherapy, vol. 22, pp. 1-8, 2021.

[16] Y. Chen, H. Chen, P. Sun, Z. Cher, S. Zhang, and Y. Lin, "Chinese herbal medicine Sijunzi decoction alleviates liver cancer cachexia through downregulating TGF-ii and IGF 1 signaling pathways," Annals of Oncology, vol. 30, 2019.

[17] X.-X. Zhang, W.-W. Chen, B. She et al., "The efficacy and safety of Jian-Wei-Qu-Tong Pills for the treatment of chronic non-atrophic gastritis (spleen and stomach qi deficiency with damp-heat stasis syndrome): study protocol for a phase II, randomized controlled trial," Trials, vol. 15, no. 1, p. 272, 2014.

[18] X.-D. Tang, B. Lu, L.-Y. Zhou et al., "Clinical practice guideline of Chinese medicine for chronic gastritis," Chinese Journal of Integrative Medicine, vol. 18, no. 1, pp. 56-71, 2012.

[19] J.-M. Chen, T.-T. Yang, T.-S. Cheng et al., "Modified Sijunzi decoction can alleviate cisplatin-induced toxicity and prolong the survival time of cachectic mice by recovering muscle atrophy," Journal of Ethnopharmacology, vol. 233, pp. 47-55, 2019.

[20] D. A. Gan, A. L. Xu, H. B. Du, and Y. A. Ye, "Chinese classical formula sijunzi decoction and chronic atrophic gastritis: evidence for treatment approach?" Evidence-based Complementary and Alternative Medicine, vol. 2017, Article ID 9012929, 9 pages, 2017.

[21] Z. Guan, J. Wu, C. Wang et al., "Investigation of the preventive effect of Sijunzi decoction on mitomycin C-induced immunotoxicity in rats by $1 \mathrm{H}$ NMR and MS-based untargeted metabolomic analysis," Journal of Ethnopharmacology, vol. 210, pp. 179-191, 2018.

[22] J. Jia, Y. Qin, L. Zhang et al., "Sijunzi decoction-treated rat serum induces apoptosis of side population cells in gastric carcinoma," Experimental and Therapeutic Medicine, vol. 15, pp. 1718-1727, 2018.

[23] A.-G. Zhao, H. L. Zhao, X. J. Jin, J. K. Yang, and L. D. Tang, "Effects of Chinese Jianpi herbs on cell apoptosis and related gene expression in human gastric cancer grafted onto nude mice," World Journal of Gastroenterology, vol. 8, no. 5, pp. 792-796, 2002.

[24] L. Chen, T. Jin, C. Ning, S. Wang, L. Wang, and J. Lin, “Antitumor and immune-modulating effect of Jiawei Sijunzi decoction in mice bearing hepatoma $\mathrm{H} 22$ tumor," Nan Fang Yi Ke Da Xue Xue Bao, vol. 39, pp. 241-248, 2019. 
[25] H.-J. Kang, M.-K. Jeong, S.-J. Park, H.-J. Jun, and H.-S. Yoo, "Efficacy and safety of Yukgunja-Tang for treating anorexia in patients with cancer," Medicine, vol. 98, no. 40, Article ID e16950, 2019.

[26] M. A. Josep, J. Francisco, T. López-Soriano Míriam, B. Angelica, S. Roberto, and B. Silvia, "The cachexia score (CASCO): a new tool for staging cachectic cancer patients," Journal of Cachexia Sarcopenia Muscle, vol. 2, no. 2, pp. 87-93, 2011.

[27] M. L. Xu, G. F. Wu, F. Huang, and J. J. Tang, "Investigation and analysis of factors influencing quality of life in elderly patients with digestive tract cancer during chemotherapy," Nursing Practice and Research, vol. 16, no. 15, pp. 13-15, 2019.

[28] Chinese Medicine Disease Diagnosis and Curative Effect Standard Editorial Committee, Chinese Criteria for Diagnosis and Efficacy of Disease, Nanjing University Press, Nanjing, China, 1994.

[29] X. Y. Zheng, Guidelines for Clinical Research of New Chinese Medicines, China Medical Science and Technology Press, Beijing, China, 2002.

[30] Y. Muliawati, H. Haroen, and L. W. Rotty, "Cancer anorexiacachexia syndrome," Acta medica Indonesiana, vol. 44, pp. 154-162, 2012.

[31] A. Laviano, M. M. Meguid, A. Inui, M. Muscaritoli, and F. Rossi-Fanelli, "Therapy Insight: cancer anorexia-cachexia syndrome-when all you can eat is yourself," Nature Clinical Practice Oncology, vol. 2, no. 3, pp. 158-165, 2005.

[32] J. B. Hopkinson, D. N. M. Wright, and C. Foster, "Management of weight loss and anorexia," Annals of Oncology, vol. 19, no. 7, pp. vii289-vii293, 2008.

[33] Z. Fan, K. Fan, Y. Gong et al., "The CRP/albumin ratio predicts survival and monitors chemotherapeutic effectiveness in patients with advanced pancreatic cancer," Cancer Management and Research, vol. Volume 11, pp. 8781-8788, 2019.

[34] J. Lu, B.-B. Xu, Z.-F. Zheng et al., "CRP/prealbumin, a novel inflammatory index for predicting recurrence after radical resection in gastric cancer patients: post hoc analysis of a randomized phase III trial," Gastric Cancer, vol. 22, no. 3, pp. 536-545, 2019.

[35] D. Unal, O. Orhan, C. Eroglu, and B. Kaplan, "Prealbumin is a more sensitive marker than albumin to assess the nutritional status in patients undergoing radiotherapy for head and neck cancer,"Współczesna Onkologia, vol. 3, pp. 276-280, 2013.

[36] T. Matsunaga, H. Miyata, K. Sugimura et al., "Prognostic significance of C-reactive protein-to-prealbumin ratio in patients with esophageal cancer," Yonago Acta Medica, vol. 63, no. 1, pp. 8-19, 2020.

[37] M. Yamamoto, H. Saito, C. Uejima et al., "Combination of serum albumin and cholinesterase levels as prognostic indicator in patients ith colorectal cancer," Anticancer Research, vol. 39, no. 2, pp. 1085-1090, 2019.

[38] S. Kimura, F. Soria, D. D’Andrea et al., "Prognostic value of serum cholinesterase in non-muscle-invasive bladder cancer," Clinical Genitourinary Cancer, vol. 16, no. 6, pp. e1123-e1132, 2018.

[39] K. E. Stanley, "Prognostic factors for survival in patients with inoperable lung Cancer2," Journal of the National Cancer Institute: Journal of the National Cancer Institute, vol. 65, pp. 25-32, 1980.

[40] M. Maltoni, A. Caraceni, C. Brunelli et al., "Prognostic factors in advanced cancer patients: evidence-based clinical recommendations-A study by the steering committee of the
European association for palliative care," Journal of Clinical Oncology, vol. 23, no. 25, pp. 6240-6248, 2005.

[41] K. T. Prasad, H. Kaur, V. Muthu, A. N. Aggarwal, D. Behera, and N. Singh, "Interconversion of two commonly used performance tools: an analysis of 5844 paired assessments in 1501 lung cancer patients," World Journal of Clinical Oncology, vol. 9, no. 7, pp. 140-147, 2018. 